\title{
3D Virtual Reality Experiments To Promote Electrical Engineering Education
}

\author{
M. Travassos Valdez, \\ C. Machado Ferreira \\ Instituto Superior de Engenharia de \\ Coimbra \\ IPC/ISEC \\ Coimbra, Portugal \\ valdez@isec.pt
}

\author{
Maria João M. Martins \\ Academia Militar \\ DCTE \& INESC-ID \\ Lisboa, Portugal \\ mariajoaomartins2@gmail.com
}

\author{
F. P. Maciel Barbosa \\ Departamento de Engenharia \\ Eletrotécnica e de Computadores \\ Faculdade de Engenharia da \\ Universidade do Porto (FEUP) and \\ INESC TEC \\ Porto, Portugal \\ fmb@fe.up.pt
}

\begin{abstract}
D Virtual Labs are the support of learning, a tool of education in the future. Learning in a 3D environment allows greater interaction with contents in a unique way as students perceive it as being really there. Simulations are being designed, a combination of real-world environment with the information supplied by devices, where measurements can be replicated and data analyzed. This study presents a virtual scenario of a Measurements and Instrumentation laboratory.
\end{abstract}

Electrical Engineering courses aim to train students to succeed in meeting the challenges within competitive and ever-changing professional contexts. In order to prepare undergraduate students for ongoing success, the courses must provide effective training in the application and development of practices, typically implemented in large projects and organizations. Developing high quality engineering course units is definitely a challenging task.

Courses can be provided remotely, but careful planning is required to design quality course units because problems may occur in meeting quality targets. The developments in information and communication technologies (ICT), including multimedia and internet-based education platforms, facilitate the creation of reliable solutions to these challenges. Studies show that a smart application of multimedia technologies and web-based education platforms can provide great results towards better quality of engineering courses. Many challenges on how to suitably approach the educational process have already been put forward. Higher Education institutions use multiple approaches to teaching and learning, combining a range of methods, including e-learning, videoconferencing, face-to-face teaching and hard-copy resources in course delivery.

The authors present a software model for engineering, which is based mainly on the Wirefusion ${ }^{\circledR}$ software, and a virtual-reality platform called VEMA. The project intends to improve the quality of teaching and learning in higher education through effective application of information technologies and the sciences of learning.

3D virtual learning environments arise as solutions to the extent that, in addition to offering features associated with communication and information sharing, they promote flexible learning spaces and moments. At the level of higher education, and in accordance with the paradigm of Bologna, virtual environments acquire special importance to allow learning beyond the classroom time and space.

Keywords-e-learning, interactive application, software packages, training and education, virtual reality prototype, $3 D$ lab internet.

\section{INTRODUCTION. E-LEARNING}

Team work, communication, project management, learning to learn and leadership are some of the requirements an engineer must meet, besides the basic technical knowledge. Engineering students also look for flexible ways to attend high quality courses and have an efficient access to the most appropriate educational facilities. On the other hand, the teaching-learning process should be student-centered, with the possibility of customizing the learning process and its outcomes.

E-learning uses the internet as a platform for learning activities, which include real-time virtual interactions between learners and educators or three dimensional environments, increasing the learning applications. The e-learning environment is considered an important resource to help traditional learning formats and is transforming the nature of higher education. Elearning platforms present several advantages as they enhance student-centered learning; students become more self-sufficient while educators take on innovative roles; the e-learning environment encourages educators to be more reflective and systematic in preparing better e-learning resources and, ultimately, provides learners with the ability to adapt to an everchanging technologically driven world. While face-to-face learning is better for ordinary discussions, contributing to an active environment, e-learning enables students to receive learning activities from educators at a distance, allows larger classes and it is easier to identify and record behaviors and errors to improve learning activities.

Today teachers are challenged to design effective learning activities that incorporate on- and off-campus resources across physical and virtual environments. Virtual Learning Environments (VLEs) provide teachers and students with a framework within which students can access learning materials and teacher support. The fundamentals of a VLE include controlled access to a curriculum divided into small sections, a system of tracking learner activity, access to learning resources, 
assessment and guidance, and a means of communication among teachers and students. Virtual laboratories are not meant to replace the need for hands-on laboratory sessions. However, these laboratories provide complementary benefits when used in addition to hands-on labs, or where the latter are unavailable. Virtual laboratories are advantageous as they give students greater flexibility of when and where to conduct experiments and allow them to complete or repeat experiments in their own time.

Distance learning (DL) uses the internet as a platform for learning activities. Students can access the online activities using individual workstations or group facilities. These activities may include: interactive quizzes, the presentation of assigned work, self and peer assessment, giving information on various issues, such as notes and deadlines, peer interaction, real time virtual interaction between students and teachers, three-dimensional images, interactive video and other applications [1-4 ] .

E-learning support technology is developing rapidly and the introduction of broadband internet allows the use of more complex information at faster rates to support an increasing array of applications for learning [5]. The learning environment is often considered an important complement to traditional forms of learning [6], and Information Technology (IT) is changing the nature of higher education [7]. Several advantages of e-learning platforms were identified: e-learning can improve student-centered learning due to the greater mobility between courses, institutions and places of study [8]; electronic assignments can be sent to students more quickly; students become more independent and self-taught while the teachers' role is related with assistance and innovation [9]; the e-learning environment encourages teachers to be better communicators and better organized in the preparation of e-learning resources [10]; it may be a more inexpensive way to offer learning activities that ensure the access to information [7]; and it provides students with the ability to adapt to an ever changing technological world [8].

Research indicates that while face-to-face learning is best for class discussion, e-learning allows students to receive high quality distance learning activities, it allows larger classes and, at the same time, it is easier to identify and record behaviors and errors so as to improve the learning activities. As an e-learning environment, it is more demanding for teachers as well [11]. To overcome some of the challenges and maximize its benefits, it is of great importance the training and mentoring of teachers and students as well as the monitoring of the students' progress.

It is also necessary an analysis of the tools and techniques currently in use, due to the high demand and complexity in building 3D virtual environments in which the speed, complexity and knowledge is crucial. It is a challenge for higher education to train new generations of engineers in the use of these tools.

\section{AVAILABLE METHODS AND SOFTWARE}

This paper proposes better practices related to the design, size and animation of 3D environments that simulate phenomena related to specific topics of electrical circuits, in order to increase the skills and knowledge by students in higher education institutions.
The goal of the project was to design and organize distributed online laboratories that allow teams of students to virtually access laboratories and to have remote collaboration in performing experiments. The aim of the project was also to create software modules for online virtual laboratories using technologies and setting up online lab workbenches. The Lab platform enables students to open laboratories for virtual access. They will use a virtual breadboard and virtual instruments to perform laboratory work from home. Tests and measurements are done using virtual instruments via the internet.

All the images of the numerous models of laboratory objects were constructed in the environment of 3Ds Max, a 3D modeling program that supports rendering images and animations. It was necessary to reduce the size of the $3 \mathrm{D}$ models and CAD files before being imported to WireFusion, while preserving visual quality. A powerful and precise polygon repair and reduction tool, the VizUp Ultimate, was used to achieve it.

The next step was to import the resources to a program used to create interactive presentations targeted to the Web. Wirefusion ${ }^{\circledR}$ was chosen to use and develop performances through visual means. WireFusion ${ }^{\circledR}$ technology allows the prompt creation of interactive 3D presentations for the internet. It is regularly used by designers, programmers, architects, creating 3D configurations, visualizing product design and architectural design. The final procedure was the introduction of all presentations on the Web or as offline presentations.

3D models are a set of polygons that can be represented geometrically in a variety of ways, in addition to having volume, color and texture. The algorithms and validation mechanisms that contribute to improve the processing, the rendering process and the mechanism used to get a good picture of the description of a 3D scene are very complex.

While the process reached a high level of realism, the main problem remains in the time spent in its execution. The objects require a lot of computer processing, and it is necessary to use methods to reduce the time spent with the rendering process. Thus, in order to combine the best way to optimize performance, it is important to choose the software, the rendering engine, and the model of the operating system.

The creation of a 3D model follows a series of steps: first, the object is constructed using polygons and other geometric elements; then, it is necessary to build the texturing and lighting because the visual reaction of the object to light is essential for the environment of a 3D model; as objects are heavy, it is essential to subject each one to a polygonal reduction; additionally, it is necessary to build the animation and rendering of the object in a scene. Once the 3D model is built, it must be able to perform all the steps to build each experiment, in order to create a 3D animation and produce a realistic virtual environment. Therefore, it is also necessary that the program used for the template is able to design a virtual camera view point, so that the scene obtains the desired effect.

3D Studio Max was used to produce the 3D model prototypes with better indicators. This software is easy to use, reliable, efficient and easy to test, flexible, reusable, and portable. However, the models could have been built in Maya or Blender (Figures 1, 2 and 3). 


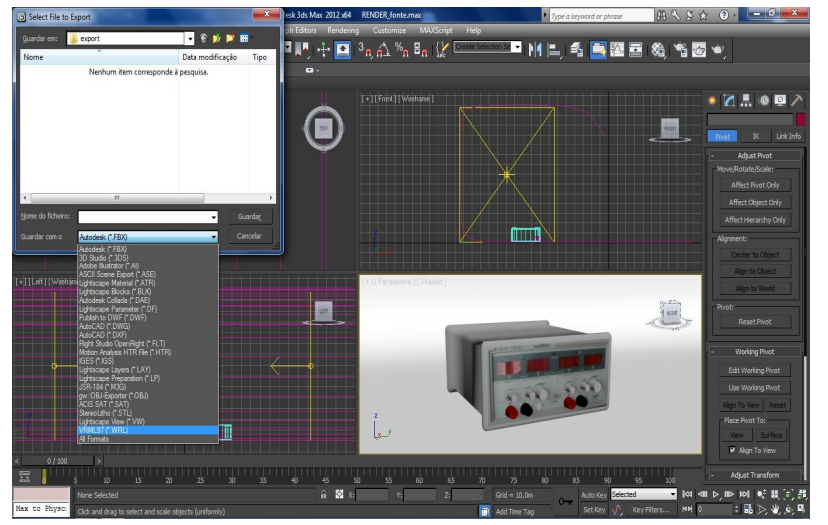

Figure 1. 3D model based on geometric shapes with textures using 3D Studio $\operatorname{Max}$

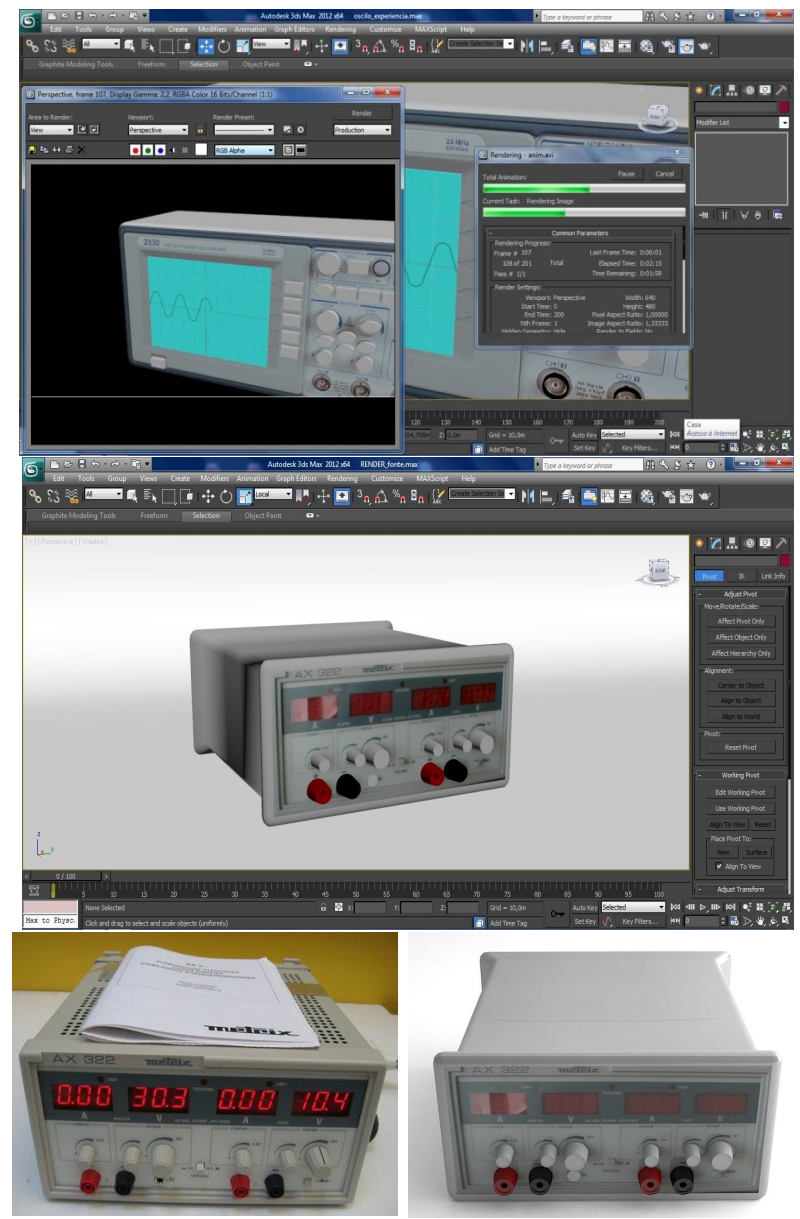

Figura 2. Power supply (real object and rendering in 3DS Max, after polygonal reduction using VizUp)

3D construction aims at replicating actual objects in the computer's memory, preserving their physical characteristics, such as size, shape and volume. To create the virtual environment, it is essential to consider all the different requirements that it will contain, such as: size, animation, effects, weather, lighting and cameras. There are several methods with different characteristics and properties to the rendering process [12].

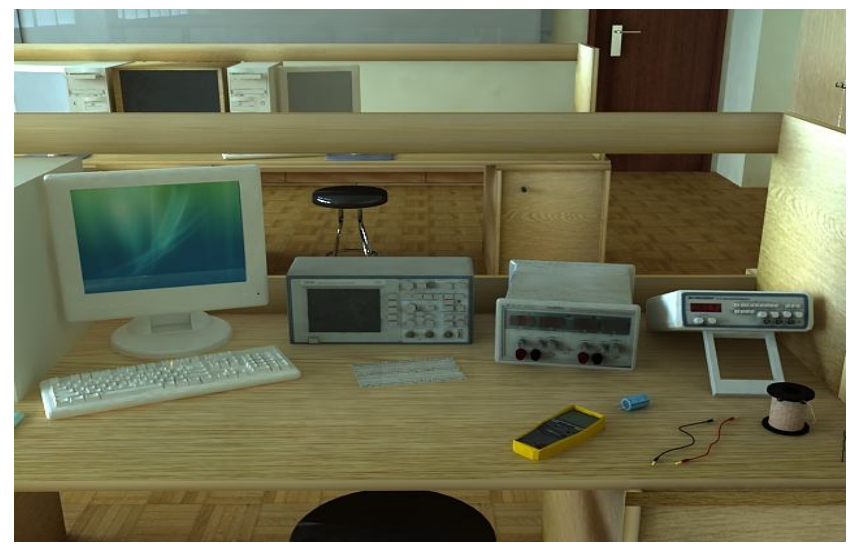

Figure 3. 3D model developed in 3D Max Studio, with elements and rendering with Vray

\section{Methodology}

A total of 5 experiments in 10 menus were conducted using the model of a 3D virtual lab environment called VEMA (Virtual Electrical MAnual). Workbench mounts were made for each experiment in order to obtain the rendering of the 3D model, according to the established parameters. Each experiment was defined by specific features, resistance values, applied voltage or current delivered; moreover, different combinations of resistors were made to develop relevant experiments (Figure 4).

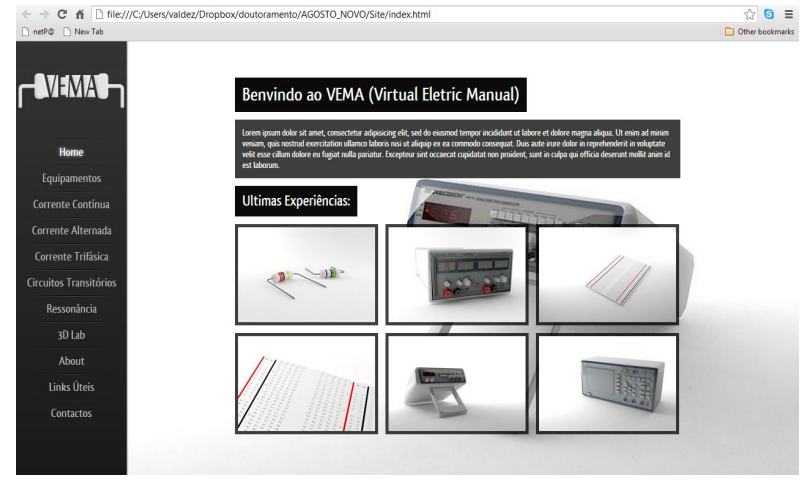

Figura 4. VEMA homepage

These sets of simulations consist of a series of experimental designs, where all tests have different parameters.

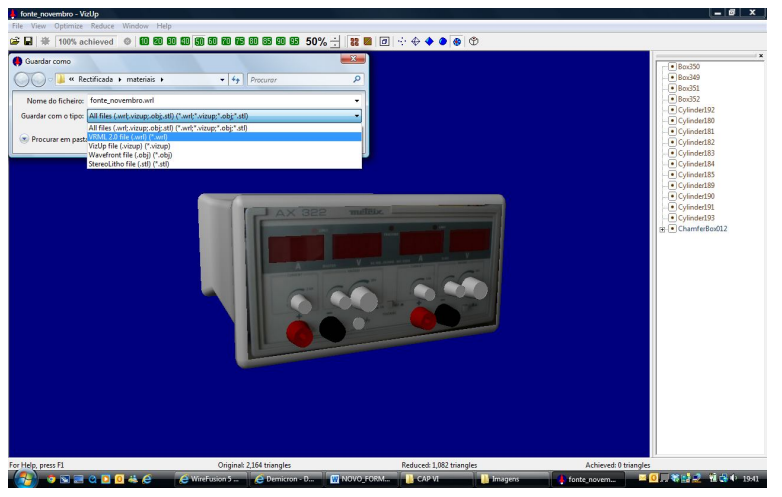

Figure 5. 3D model developed in 3D Max Studio, with the elements and rendering using Vray 
Figure 5 shows the use of the MentalRay rendering engine to generate a processing technique.

Based on comparisons generated in this study, it is essential to use a standard process to understand all the steps necessary for the ideal development of the 3D environment and to generate better applications with the best tools. A process that helps generate these paths is proposed, as shown in Figure 6.

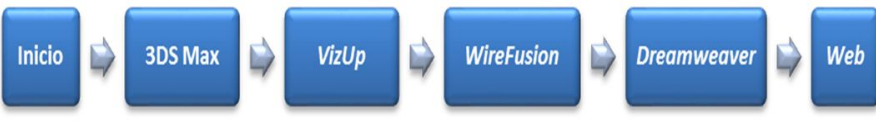

Figure 6. 3D virtual application development model

The first step is to create all the necessary resources: 3D models, images, animation, sound, etc.

The next step is to upload these 3D models to a polygon reduction program. VizUp was used, which allowed the reduction and conversion of models without damaging the representation of the object. It reduces polygons of entities in VRML format, and makes it possible to import complex entities, without sacrificing the speed of the computer and accelerating the rendering process [13]. This was important for the publication of contents on the internet.

The third step is to import these resources to WireFusion 5.0 (R) and develop presentations through visual means [14]. This software is the simulation scenario editor. In this editor, all entities and their respective behaviors, made in 3DS Max within the scenarios (maps), are imported; furthermore, the editor allows the attachment of lighting, textures and other aspects related to the creation of virtual sets.

The fourth and final step is the production of interactive contents using HTML5, CSS3 and Javascript with the aim of creating standard presentations that may be used on the web, online or offline [15-16].

\section{CONCLUSION}

The use of 3D models has become a common practice. Due to the large number of tasks to be processed, advanced computer equipment is required. Furthermore, the integration of new technologies allows the work to be performed without affecting the processing speed and/or the quality of results. As the creation of 3D environments for higher capacity computers involves time and resources, this is a factor that will cause many institutions to restrict their expectations in generating more interesting projects.

Finally, due to its usefulness in higher education, it is intended that 3D virtual labs are to be developed along with other learning materials for better performance in the teaching and learning of higher-level processes.

A desirable scenario will have courses and/or curricular units given in distance learning mode, allowing students to access practical laboratory work from home or from work.

With WireFusion, it is no longer necessary to plug-in to operate 3D presentations. The focus was transferred to the creation of 3D presentations. Presentations in WireFusion can, therefore, be used for both e-learning and computer-based training (CBT-computer based training) in a friendly and intuitive manner, in order to create educational applications.

Teaching management platforms based on e-learning methodologies can contribute to the creation of new learning environments that foster interaction and collaboration and, generally, facilitate teaching and learning.

The simulation on a computer is an efficient technique for practical work applications. These simulations minimize the concerns of teachers regarding time, costs or risks that involve the choice of inappropriate, inefficient, or potentially dangerous experimental strategies. The conclusions show the benefits of integrating this innovative model in an engineering course. Not only does this approach reinforce the acquisition of current engineering practices, but also opens new possibilities in the design of training courses.

\section{REFERENCES}

[1] K. Bijlani, P. Manoj, P. Venkat Rangan. "A Framework for Interactive eLearning in a Virtual World”. BIS (Workshops), pp 177-187. 2008.

[2] M. Auer, Pester A., Ursutiu, D., Samoila C., Distributed virtual and remote labs in engineering, IEEE International Conference on Industrial Technology, vol. 2, pp. 1208-1213. 2003.

[3] D.Gillies, Student perspectives on videoconferencing in teacher education at a distance. Distance Education, 29(1), pp 107-118. 2008.

[4] E. Stacey and F. A Wiesenberg. "Study of face-to-face and online teaching philosophies in Canada and Australia”. Journal of Distance Education. 22(1), pp 19-40. 2007.

[5] E. J. Burge, C. C. Gibson and T. Gibson. "Flexible pedagogy, flexible practice: Notes from the trenches of distance education". s.l.:Athabasca University Press. 2012.

[6] G. J. Hwang and H. F. Chang. "A formative assessment-based mobile learning approach to improving the learning attitudes and achievements of students”. Computers \& Education, Volume 56, pp. 1023-1031. 2011.

[7] C. Normand, A. Littlejohn, and I. Falconer. "A model for effective implementation of flexible programme delivery". Innovations in Education and Teaching International, 45(1), pp. 25-36. 2008.

[8] M. A. Kamba. "Problems, Challenges and Benefits of Implementing Elearning in Nigerian Universities: An Empirical Study". doi:10.3991/ijet.v4i1.653. iJET 4(1). 2009.

[9] A. K. Manir. "The Internet as tool for Interactive Learning, Teaching and Research". International Journal of Emerging Technologies in Learning 2 (3). 2007.

[10] K. Purcell et al., "How Teachers Are Using Technology at Home and in Their Classrooms". (Washington, DC: Pew Research Center's Internet \& American Life Project, 2013.

[11] J. L. Bishop and M. L. Verleger. "The Flipped Classroom: A Survey of Research". Paper prepared for the Association for Engineering Education 120th Conference, Atlanta, GA, June 2013.

[12] 3Ds Max. Autodesk® 3Ds Max Design®. Available from: http://usa.autodesk.com/3ds-max/\#. [Accessed June 10, 2012].

[13] VizUp software company - Optimize 3D models. [on line] Disponível em: http://www.vizup.com/index.html,

[14] Disponível em: http://download.cnet.com/WireFusion-Free-Edition/ 3000-6677_4-10808107.html. Acessível em 27 junho 2010.

[15] M. M., Travassos Valdez, C. M., Machado Ferreira and F. P. Maciel Barbosa," "Software Packages to Support Electrical Engineering Virtual Lab". International Journal on Emerging Technologies in Learning, iJOE2012. ISSN: 1861-2121. Volume 8, Special Issue 2: exp.at'11, pp. 19-23, Special Focus Pa.pers. 2012.

[16] M. T. Valdez, C M. Ferreira, F. P. Maciel Barbosa. "Software Packages to Support Electrical Engineering Virtual Lab," International Journal of Online Engineering (iJOE), pp. 19-23. 2012. 